\title{
IMPROVING ROBUSTNESS AGAINST ELECTRODE SHIFT OF SEMG BASED HAND GESTURE RECOGNITION USING ONLINE SEMI-SUPERVISED LEARNING
}

\author{
QIU XIA LI ${ }^{1}$, PATRICK P. K. CHAN ${ }^{1}$, DALIN ZHOU ${ }^{2}$, YINFENG FANG ${ }^{2}$, HONGHAI LIU ${ }^{2}$, DANIEL S. YEUNG \\ ${ }^{1}$ School of Computer Science and Engineering, South China University of Technology, Guangzhou, China \\ ${ }^{2}$ Portsmouth University, Portsmouth, United Kingdom \\ E-MAIL: sunshinelqx@outlook.com, patrickchan@scut.edu.cn,yinfeng.fang@port.ac.uk, dalin.zhou@port.ac.uk, \\ honghai.liu@icloud.com, danyeung@ieee.org
}

\begin{abstract}
:
Electrode shift of a prosthetic device is one of most challengeable problems in surface Electromyography (sEMG) based hand gesture recognition. Electrode shift is usually caused by repositioning, donning or doffing of a prosthetic device. Accuracy of gesture recognition may significantly drop since a pattern of collected signals may change after electrode shift. Although re-training a recognition system after every reposition is able to maintain accurate recognition, collecting labeled samples is inconvenient to users. In this paper, we apply an online semi-supervised learning in which a classifier is trained with a small amount of labeled samples and then is updated with unlabeled samples online to hand gesture recognition. A well-known online semi-supervised learning algorithm, online multi-channel semi-supervised growing neural gas (OSSMGNG) algorithm, is used in this preliminary study. OSSMGNG is compared with an intuitive method which learns from the initial label training set only in experiments. The data is collected from able-bodied individuals across three days for experiments. The results indicate OSSMGNG achieves a higher classification accuracy than others. It suggests that the online semi-supervised learning algorithm enhances robustness of hand gesture identification against electrode shift.
\end{abstract}

\section{Keywords:}

Online Semi-supervised learning; Growing neural gas; Hand gesture recognition; Electrode shift

\section{Introduction}

The surface electromyography (sEMG) is an important type of biological signal generated by activities of musculature. Many studies have showed that sEMG recognition achieves satisfying performance in myoelectric control systems [1-4]. Hand gesture recognition determines sEMG signals of arm muscles to control a hand prosthetic device. Recognizing gesture accurately is the important problem in hand gesture recognition.

Many pattern recognition techniques have been applied to sEMG signal recognition in recent decades. A number of research works [4-7] achieved accurate performance in hand gesture recognition using support vector machine (SVM) and linear discriminant analysis (LDA). Most studies assume there is an ideal environment. However, there are many factors which cause sEMG nonstationary in daily use. Thus robustness of hand gesture recognition systems should be considered.

Electrode shift is one of most challengeable problems in sEMG based hand gesture recognition. The movement of electrode is usually caused by donning and doffing of prosthesis, and muscle fatigue [4], [8]. Many studies showed that accuracy of the recognition significantly [8-11] drops due to electrode shift since the distribution of collected signals changes. Although re-training the system [11], [12] can maintain satisfying performance, a training process requires labelled samples. Sample collection is inconvenient to users. It is impractical in daily use.

In this paper, we apply an online semi-supervised learning to hand gesture recognition in order to reduce influence of electrode shift. A classifier is trained with a small amount of labeled samples and then is updated with unlabeled samples online in online semi-supervised learning. Online multi-channel semi-supervised growing neural gas (OSSMGNG) [13], which is one of well-known methods, is used in this paper. OSSMGNG contains two layers: the first layer is the category layer, and the other layer is the topological layer of associated classification. The category layer stores the hand gestures labels. Each gesture has its own topological layer, which is a cluster of neurons that learned from the input data space. Labeled data is used to train the initial GNG classifier. GNG is then updated by unlabeled samples and their predicted label provided by distance-weighted k-nearest neighbor 
algorithm (DW-KNN). OSSMGNG updates the classifier using unlabeled samples. As unlabeled samples are collected automatically during daily use, no additional input is required from users. Moreover, the system is able to learn new distribution of sEMG after electrode shift. Therefore, it is expected that the performance of OSSMGNG should be better than the traditional supervised learning.

The rest of paper is arranged as follows. Section 2 reviews the related research work of electrode shifts and semi-supervised learning. In Section 3, we describe OSSMGNG algorithm in detail. Experimental setting is introduced and results are discussed in Section 4. Section 5 concludes this study.

\section{Related work}

\subsection{Electrode Shift}

Myoelectric control systems apply sEMG signals to recognize hand gestures. sEMG is surface action potentials, and is sensitive to electrode repositioning. Electrode position change may be due to donning and doffing of prosthesis, or muscle fatigue in day-to-day [11]. Electrode shift has negative effect on the performance of a myoelectric control system.

Several kinds of methods which reduce the influence of electrode shift were proposed recently. From the signal point of view, a robust sEMG electrodes configuration [14] was proposed. This configuration measures EMG signals from all arm muscles to resolve crosstalk. Another study [15] improved the robustness by increasing inter-electrode distance from 2 to $4 \mathrm{~cm}$. Moreover, electrode configuration that combined of longitudinal and transverse channels is devised to capture more stable myoelectric signals.

On the other hands, some methods [1], [12] focus on a robust learning model which requires re-training after electrode shift. Since the distribution of data collected by electrode is changed due to the shift problem, accuracy of the recognition systems drops. Re-training process makes the recognition systems learn the new distribution. Although these methods achieve satisfying results in hand gesture recognition, collecting labelled samples from users is inconvenience. A study [11] devised the re-training process which requires less required labeled samples. However, although the duration of data collection is shorten, the setup procedure may still be complicated. Even worse, data collection may be impractical when a data collection system is not accessible.
Online semi-supervised learning builds a system by learning from not only labeled but also unlabeled samples. A classifier is trained firstly using with labeled (and unlabeled) samples. Different from the offline one, the classifiers should be updated continually using unlabeled samples in online semi-supervised learning. One of the advantages is that labelled samples are only required in the initial training since obtaining label can be expensive, time-consuming or even impractical. Due to this flexibility, online semi-supervised learning has been applied to many applications, e.g. human activity recognition and human daily-life activity recognition [13], face recognition [16] and tracking applications [17], with satisfying performance.

Most of online semi-supervised learning methods are extended from offline ones. There are five main semi-supervised learning methods, including generative model, co-training methods, self-training methods, graph-based learning methods and low-density separation methods [18], [19]. Generative models may be the oldest semi-supervised learning method. It assumes that data samples follow a mixture distribution in which the mixture components can be identified from large amount of unlabeled data [20]. Co-training methods split features into independent two sub-feature sets which contains enough information for training a classifier with satisfying performance. Two classifiers are trained with labeled data separately. A classifier is retrained using a unlabeled sample and its labeled predicted by another classifier [21]. A classifier is firstly trained with small amounts of labeled data in self-training method. The classifier then predicts labels for all unlabeled data with a confidence level. Samples with the most confident values will be included in the training set. These iterative procedures stop until the unlabeled set is null. [18]. Graph-based learning methods construct a graph containing labeled and unlabeled data as nodes. The edges represent the similarity of nodes. Graph-based method makes a decision function based on a graph by optimizing a loss function and a regularizer, for example, Gaussian Random Fields and Harmonic Functions, Manifold Regularization and Mincut [20]. Low-density separation methods use unlabeled data to find a maximum margin linear boundary away from high density regions. Transductive support vector machines (TSVM) is a typical extension of standard support vector machines with unlabeled data [22]. 


\section{Online semi-supervised growing neural gas (OSSMGNG) classifier [10] for hand gesture recognition}

In this section, OSSMGNG is firstly introduced. Then we will describe how it can be applied to hand gesture recognition to reduce influence of electrode shift.

\subsection{OSSMGNG}

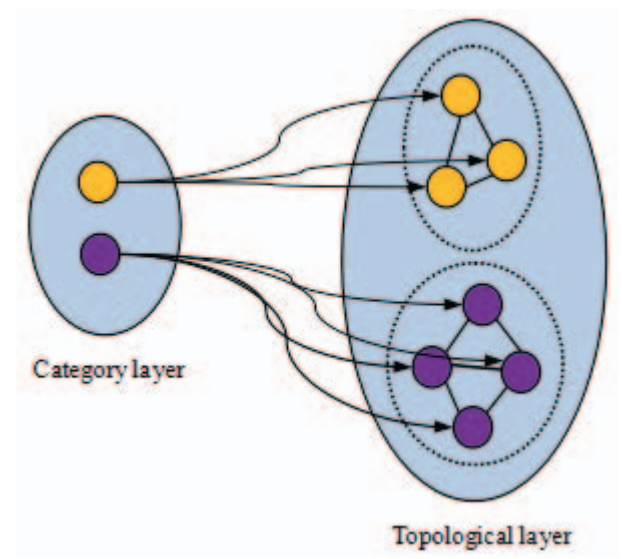

FIGURE 1. Architecture of OSSMGNG with two classes

OSSMGNG proposed by Parham Nooralishahi et al. [13] is an incremental learning method based on growing neural gas. This method improves the performance of a classifier by using a small amount of labeled data and a large amount of unlabeled data. The architecture of OSSMGNG can be illustrated by two layers. The first layer named category layer consists of class nodes. A class node represents a specific category of the samples. The topological structure of each node is stored in the second layer. The structure is built according to Growing Neural Gas (GNG) algorithm. The architecture is shown in Figure 1.

Topological structure of each class is firstly created in OSSMGNG according to GNG algorithm using labeled samples. GNG algorithm aims to represent a dataset using topological structure, which is a digraph, created by a number of nodes. Nodes are adjusted and added to the structure iteratively. Two samples are randomly selected as the nodes and are connected initially. The age of their edge and their error values are set to 0 . For a sample $x$, all edges connected to the closet node $\left(s_{0}\right)$ of $x$ are incremented by 1 . Then, $s_{0}$ and its nearest neighbor node $\left(s_{n}\right)$ are moved closer to $x$ according to

$$
s_{0}{ }^{\prime} \leftarrow s_{0+} e_{0} \times \operatorname{dist}\left(x, s_{0}\right) \text {, and }
$$

$$
s_{n}{ }^{\prime} \leftarrow s_{n+} e_{n} \times \operatorname{dist}\left(x, s_{n}\right)
$$

where $e_{0}$ and $e_{\mathrm{n}}$ are update parameters, and $s_{0}$ ' and $s_{\mathrm{n}}$ ' are the updated position of of $s_{0}$ and $s_{\mathrm{n}}$ respectively. dist is the distance function. The error value of $s_{0}$ denoted by $e r r_{\mathrm{s}}$ is updated according to

$$
\operatorname{err}_{\mathrm{s}}^{\prime} \leftarrow \operatorname{err}_{\mathrm{s}}-\left(\text { error }_{\mathrm{s}} \times \beta\right)
$$

where $\beta$ is the error update parameter of all nodes.

After each $\lambda$ iterations, the structure is expanded by adding a new node. The node with the maximum error $\left(s_{\max }\right)$, and also its neighbor node with the largest error $\left(s_{\mathrm{e}}\right)$ are selected. This indicates that the structure cannot represent the dataset well in this region and more nodes are needed. Therefore, the new node is added to the middle location of $s_{\max }$ and $s_{\mathrm{e}}$, and connect to them. The error of the new node is set to 0 , while the error values of $s_{\max }$ and $s_{\mathrm{e}}$ are reduced by.

$$
\begin{gathered}
\operatorname{err}_{\mathrm{s}_{\text {max }}}^{\prime} \leftarrow \operatorname{err}_{\mathrm{s}_{\max }}-\left(\operatorname{err}_{\mathrm{s}_{\max }} \times \alpha\right), \text { and } \\
\operatorname{err}_{\mathrm{s}_{\mathrm{e}}}^{\prime} \leftarrow \operatorname{err}_{\mathrm{s}_{\mathrm{e}}}-\left(\operatorname{err}_{\mathrm{s}_{\mathrm{e}}} \times \alpha\right)
\end{gathered}
$$

where $\alpha$ is the error update parameter of $s_{\max }$ and $s_{\mathrm{e}}$.

After the topological structures are created, they can classify an unseen sample based on distance-weighted k-nearest neighbor algorithm (DWKNN) [23]. DWKNN uses the dual distance-weighted function to find $\mathrm{k}$ closest nodes to the unseen sample. A weight is assigned to each closest node according to the distance between the node and the unseen sample, i.e. a smaller weight is assign to a node with a longer distance; vice versa. A label of an unseen sample is determined by the weighted majority. The unseen sample is then used to update the topological structure of its predicted class according to GNG algorithm. As a result, the system is updated continually with unlabeled samples in OSSMGNG.

The topological structures can be simplified according to competitive Hebbian learning (CHL) rule [24]. A connections with age values larger than threshold and a node without connections will also be removed in order to increase time and space efficiency.

\subsection{OSSMGNG for hand gesture recognition}

sEMG based hand gesture recognition is a long-term and online task. Samples with labels are collected from a user for training a system initially. Unlabeled samples can be obtained collected automatically during daily use. We would like to obtain useful information from unlabeled samples by OSSMGNG to avoid labelled sample collection.

Topological structure of each gesture is firstly created according to the labelled samples collected from a user. During daily use, unlabeled samples are collected and update the system. For the updating rules in (1) and (2), Euclidean distance instead of multi-channel dynamic time 
warping is used since the dimension of features in each sample is equal in hand gesture application.

As OSSMGNG learns from the latest sEMG signals continuously, it is expected that the performance of OSSMGNG is better than the one trained with the initial labeled samples and no further update. Accuracy of OSSMGNG is evaluated experimentally in next section.

\section{Experiments}

We evaluate the performance of OSSMGNG algorithm for hand gesture recognition against electrode shift using two datasets, which are collected from able-bodied individuals across 3 days. The results are compared with ones of the traditional classification techniques.

\subsection{Dataset description}

Samples of two datasets are also collected from able-bodied individuals across 3 days (i.e. day 1, 2 and 3). An individual wear the prosthetic device every day, i.e. electrode shift occurs on Day 2 and 3. We collect 100 samples for each of ten muscles motions including Extensor Carpi Uinaris (ECU), Extensor Digitorum (ED), Extensor Carpi Radiails Brevis (ECRB), Extensor Digiti Minimi (EDM), Flexor Carpi Ulnaris (FCU), Flexor Digitorum Profundus (FDP), Abductor Pollicis Longus (APL), Extensor Pollicis Brevis (EPB), Extensor Pollicis Longus (EPL) and Extensor Indicis (EE) for each individual each day. Totally, there are 300 samples of each class per day in dataset 2. In this experimental, Wave Length (WL) [25] is chosen as a feature. Dataset 1 is collected by using traditional sEMG electrodes configuration and dataset 2 is collected by paper [14] using new sEMG electrodes configuration.

\subsection{Experimental Setting}

This experiment aims to evaluate if using unlabeled samples can help the hand gesture recognition against electrode shift. All samples in Day 1 are treated as the labelled set, while the samples in Day 2 and 3 are unlabeled. In this section, we evaluate three methods including 1) OSSMGNG: KNN is trained by samples in Day 1 and is updated continually by unlabeled samples in Day 2 and 3,2) $\mathrm{K}$-nearest Neighbor algorithm $(\mathrm{KNN})$ : $\mathrm{KNN}$ will not be updated after training by using data in day 1-) K-nearest Neighbor algorithm with feedback (KNN_Feedback): KNN is updated at the end of a day using the labeled sample. Classification accuracy of these methods are measured on samples in Day 2 and 3.
The parameters of OSSMGNG algorithm is determined for each person in each dataset through a 5-fold cross-validation to minimize the classification error. For dataset 1 , the parameters are $\max _{\text {age }}=60, \lambda=60$, $\epsilon_{0}=0.01, \epsilon_{n}=0.01, \alpha=0.5, \beta=0.995$ for individual 1 , and $\max _{a g e}=60, \lambda=60, \epsilon_{0}=0.01, \epsilon_{n}=0.01, \alpha=0.5$, $\beta=0.995$ for individual 2 and 3; For dataset 2, the parameters are $\max _{a g e}=80, \lambda=200, \epsilon_{0}=0.0321, \epsilon_{n}=$ $0.02, \alpha=0.5, \beta=0.995$ for all individuals. We choose $\mathrm{k}$ $=5$ for the classifiers of all three methods.

\subsection{Experimental Result}

Table 1 shows the average classification accuracy of the hand gesture methods among three different individuals on dataset 1. OSSMGNG achieves significantly higher accuracy than KNN. It confirms that unlabeled samples are useful in reducing the influence of electrode shift in hand gesture recognition by online semi-supervised learning. The accuracy of KNN_Feedback is $70.6 \%$ in Day 3, which is slightly higher than $68.8 \%$ achieved by OSSMGNG. However, KNN_Feedback requires the input from users. It also make users inconvenient.

Table 2 shows the average percentage of classification accuracy of the three methods on dataset 2. The general performance of all methods on this dataset is dramatically better than one on dataset 1 . This is because the influence of electrode shift is minimized during the data collection. In this dataset, we can see OSSMGNG and KNN do not have significantly different accuracies. This is because the factor of electrode shift barely affects the recognition.

Accuracies of ten hand gesture motions are shown in Figures 2 and 3. The performance of OSSMGNG on the classes FCU and APL are unstable. For samples in APL, OSSMGNG is worse in day 2 but better in day 3 than other two methods. In contrast, accuracy of OSSMGNG is higher day 2 but lower in day 3 than other two methods. There are no significantly change on accuracies of all methods on samples in classes ECRB, EDM and ECU. It may indicate that electrode shift does not affect these classes obviously.

TABLE 1. Classification accuracy of the three methods on dataset 1

\begin{tabular}{lll}
\hline & Day 2 & Day 3 \\
\hline OSSMGNG & $62.8 \%$ & $68.8 \%$ \\
\hline KNN & $57.9 \%$ & $62.8 \%$ \\
\hline KNN_Feedback & $57.9 \%$ & $70.6 \%$ \\
\hline
\end{tabular}


TABLE 2. Classification accuracy for the three methods on dataset 2

\begin{tabular}{lll}
\hline & Day 2 & Day 3 \\
\hline OSSMGNG & $91.3 \%$ & $91.6 \%$ \\
\hline KNN & $90.0 \%$ & $91.4 \%$ \\
\hline KNN_Feedback & $90.0 \%$ & $96.8 \%$ \\
\hline
\end{tabular}

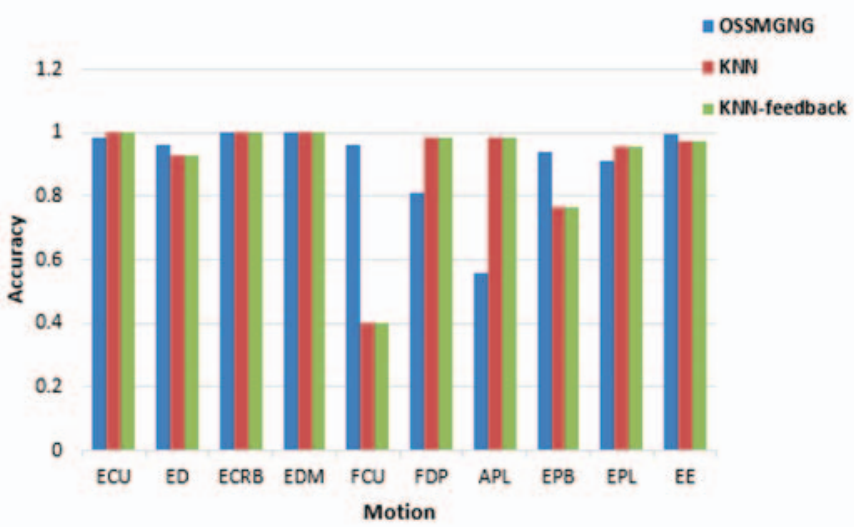

FIGURE 2. Classification accuracy of ten hand gesture motions in Day 2

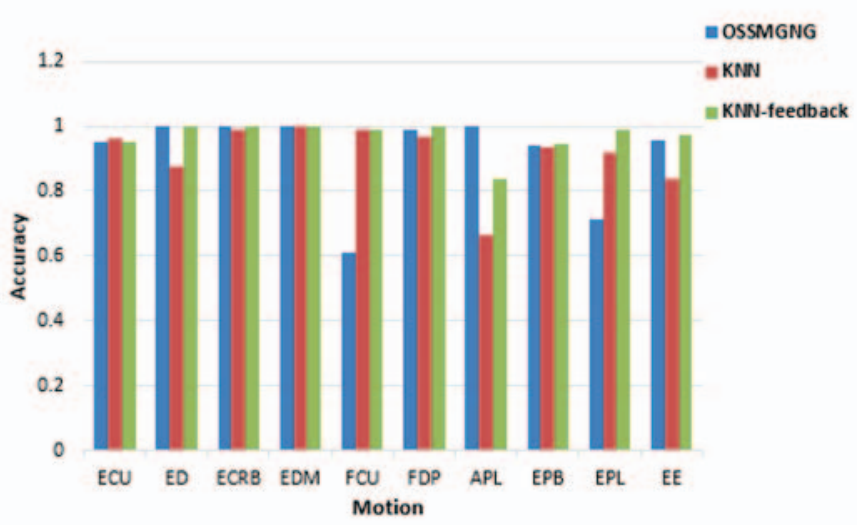

FIGURE 3. Classification accuracy of ten hand gesture motions in Day 3

\section{Conclusions}

Electrode shift reduces classification accuracy of sEMG based hand gesture recognition. Although re-training the systems with labeled samples can maintain the performance, it is very inconvenience to a user. To avoid collection of labeled samples, this paper investigates whether an online semi-supervised learning is helpful in reducing influence of electrode shift in hand gesture recognition. We apply one of well know method, OSSMGNG algorithm, in this paper. The topological structures of samples are created initially using labeled samples and then are updated by unlabeled samples. It improves accuracy of a classifier with both labeled and unlabeled data. The experiments are carried out using two datasets from able-bodied individuals across three days. The results suggest that OSSMGNG achieves higher accuracy than K-nearest Neighbor algorithm. It indicates that unlabeled samples contain useful information to improve accuracy of recognition by online semi-supervised learning. However, OSSMGNG does not perform well on samples in all classes. In future, the change of sample distribution can be considered to improve stability and accuracy of online semi-supervised learning.

\section{Acknowledgements}

This work is supported by "the Fundamental Research Funds for the Central Universities" (2015ZZ092).

\section{References}

[1] Clement R G E, Bugler K E, Oliver C W. Bionic prosthetic hands: A review of present technology and future aspirations $[\mathrm{J}]$. The surgeon, 2011, 9(6): 336-340.

[2] Erik Scheme MSc P E, Kevin Englehart PhD P E. Electromyogram pattern recognition for control of powered upper-limb prostheses: State of the art and challenges for clinical use [J]. Journal of rehabilitation research and development, 2011, 48(6): 643.

[3] Oskoei M A, Hu H. Myoelectric control systems-A survey $[\mathrm{J}]$. Biomedical Signal Processing and Control, 2007, 2(4): 275-294.

[4] Farina D, Jiang N, Rehbaum H, et al. The extraction of neural information from the surface EMG for the control of upper-limb prostheses: emerging avenues and challenges $[\mathrm{J}]$. Neural Systems and Rehabilitation Engineering, IEEE Transactions on, 2014, 22(4): 797-809.

[5] Lucas M F, Gaufriau A, Pascual S, et al. Multi-channel surface EMG classification using support vector machines and signal-based wavelet optimization [J]. Biomedical Signal Processing and Control, 2008, 3(2): 169-174.

[6] Khokhar Z O, Xiao Z G, Menon C. Surface EMG pattern recognition for real-time control of a wrist 
exoskeleton [J]. Biomedical engineering online, 2010, 9(1): 1.

[7] Young A J, Smith L H, Rouse E J, et al. Classification of simultaneous movements using surface EMG pattern recognition $[\mathrm{J}]$. IEEE Transactions on Biomedical Engineering, 2013, 60(5): 1250-1258.

[8] Young A J, Hargrove L J, Kuiken T A. The effects of electrode size and orientation on the sensitivity of myoelectric pattern recognition systems to electrode shift [J]. IEEE Transactions on Biomedical Engineering, 2011, 58(9): 2537-2544.

[9] Amsüss S, Paredes L P, Rudigkeit N, et al. Long term stability of surface EMG pattern classification for prosthetic control [C]//2013 35th Annual International Conference of the IEEE Engineering in Medicine and Biology Society (EMBC). IEEE, 2013: 3622-3625.

[10] Fougner A, Scheme E, Chan A D C, et al. Resolving the limb position effect in myoelectric pattern recognition[J]. IEEE Transactions on Neural Systems and Rehabilitation Engineering, 2011, 19(6): 644-651

[11] Vidovic M M C, Paredes L P, Hwang H J, et al. Covariate shift adaptation in EMG pattern recognition for prosthetic device control [C]//2014 36th Annual International Conference of the IEEE Engineering in Medicine and Biology Society. IEEE, 2014: 4370-4373.

[12] Chicoine C L, Simon A M, Hargrove L J. Prosthesis-guided training of pattern recognition-controlled myoelectric prosthesis $[C] / / 2012$ Annual International Conference of the IEEE Engineering in Medicine and Biology Society. IEEE, 2012: 1876-1879.

[13] Nooralishahi P, Seera M, Loo C K. Online semi-supervised multi-channel time series classifier based on growing neural gas $[\mathrm{J}]$. Neural Computing and Applications, 2016: 1-15.

[14] Fang Y, Liu H. Robust sEMG electrodes configuration for pattern recognition based prosthesis control $[C] / /$ Systems, Man and Cybernetics (SMC), 2014 IEEE International Conference on. IEEE, 2014: 2210-2215.

[15] Young A J, Hargrove L J, Kuiken T A. Improving myoelectric pattern recognition robustness to electrode shift by changing interelectrode distance and electrode configuration $[\mathrm{J}]$. IEEE Transactions on Biomedical Engineering, 2012, 59(3): 645-652.

[16] Zaki S M, Yin H. A semi-supervised learning algorithm for growing neural gas in face recognition [J]. Journal of Mathematical Modelling and Algorithms, 2008, 7(4): 425-435.

[17] Grabner H, Leistner C, Bischof H. Semi-supervised on-line boosting for robust tracking $[\mathrm{C}] / /$ European conference on computer vision. Springer Berlin Heidelberg, 2008: 234-247.

[18] Chapelle O, Scholkopf B, Zien A. Semi-Supervised Learning (Chapelle, O. et al., Eds.; 2006) [Book reviews] [J]. IEEE Transactions on Neural Networks, 2009, 20(3): 542-542.

[19] Amini M R, Usunier N. Semi-supervised learning $[\mathrm{M}] / /$ Learning with Partially Labeled and Interdependent Data. Springer International Publishing, 2015: 33-61.

[20] Zhu X. Semi-supervised learning literature survey [J]. 2005.

[21] Li K, Zhang J, Xu H, et al. A semi-supervised extreme learning machine method based on co-training $[\mathrm{J}]$. J Comput Inf Syst, 2013, 9(1): 207-214.

[22] Esposito G, Martin M. A randomized algorithm for the exact solution of transductive support vector machines [J]. Applied Artificial Intelligence, 2015, 29(5): 459-479.

[23] Gou J, Du L, Zhang Y, et al. A new distance-weighted k-nearest neighbor classifier [J]. J. Inf. Comput. Sci, 2012, 9(6): 1429-1436.

[24] Martinetz T, Schulten K. Topology representing networks [J]. Neural Networks, 1994, 7(3): 507-522.

[25] Rafiee J, Rafiee M A, Yavari F, et al. Feature extraction of forearm EMG signals for prosthetics [J]. Expert Systems with Applications, 2011, 38(4): 4058-4067. 